\title{
Energy Efficient Routing Protocol with Improved Clustering Strategies for Homogeneous Wireless Sensor Networks
}

\author{
T. Venu Madhav \\ Research Scholar, \\ Dept. of ECE \\ National Institute of Technology, \\ Warangal, India
}

\author{
N V S N Sarma \\ Professor, \\ Dept. of ECE \\ National Institute of Technology, \\ Warangal, India
}

\begin{abstract}
A new energy aware routing protocol to optimize energy consumption and prolong network lifetime for Wireless Sensor Networks (WSNs) had been proposed in this paper. Cluster based routing techniques like the conventional Low Energy Adaptive Clustering Hierarchy $(\mathrm{LEACH})$ are used to achieve scalable solutions and extend the network lifetime until the last node dies. Improved Energy Efficient LEACH (IMP-EEL) has been proposed in this work and compared with the other existing algorithms like LEACH, Residual Energy LEACH (RES-EL) and Distributed Residual Energy LEACH (DIS-RES-EL). The proposed algorithm (IMP-RESEL) outperformed all the other algorithms in extending network lifetime, network stability, sending aggregated packets to Cluster heads (CHs) and to Base station(BS) and $\mathrm{CH}$ formation during their entire lifetime considered. With optimum routing established within the network as per the newly proposed clustering threshold, IMP-EEL has significantly reduced energy consumption and maintained $72 \%$ more energy efficiency than the LEACH homogeneous system.
\end{abstract}

\section{General Terms}

Wireless Sensor Networks, clustering, energy efficiency, homogeneous network.

\section{Keywords}

LEACH protocol, network lifetime, cluster head selection, network stability, homogeneous, routing protocols, wireless sensor networks.

\section{INTRODUCTION}

A wireless sensor network (WSN) is a wireless network consisting of spatially distributed autonomous devices using sensors to monitor physical or environmental conditions. These sensors detect the events collect, store and process data, transmit the sensed information to the interested observers [1]. With the advancement of several wireless devices and electronics, operation of sensor nodes working properly for long run of time in WSNs has received increasing attention. WSNs are autonomous ad hoc networks designed for some potential applications in environmental monitoring, surveillance, military, health, security, and so on. [2].

Sensor network is an interdisciplinary research process that draws contributions from signal processing, networking and protocols, databases and information management, distributed algorithms and embedded systems and architecture. The main purpose of WSN is to serve as an interface to the real world, providing physical information such as temperature, light, humidity, radiation stress pattern etc. to a computer system. Because the most challenging issue in sensor networks is energy resource. Most of the nodes are battery operated and cannot be recharged. Many research efforts are aiming to improve the energy efficiency from different routing protocols [3]. It is desirable to develop energy-efficient processing techniques across all levels of the protocol stack and at the same time, minimize message passing for network control and coordination among the nodes. Hence, the sensors must be wisely managed in order to extend the lifetime of the network. Most tasks require the combined effort of multiple network nodes, requiring protocols to provide coordination, efficient local exchange of information, and possibly, hierarchical operation.

Developing clustering algorithms provide an efficient way to save energy for static sensor networks [4-7]. Clustering has three significant differences from conventional clustering schemes. First, data compression in the form of distributed source coding is applied within a cluster in order to reduce the transmission of number of packets $[8,9]$. Second, the datacentric property makes an identity for a sensor node obsolete. In fact, the observers or users are often interested in phenomena occurring in a specified area, rather than in an individual sensor node [10]. Third, randomized rotation of cluster heads ensures balanced energy consumption to the total network.

\section{RELATED WORK}

Energy-aware routing [11] avoids the increase in energy consumption for communication to transmit or receive information among the nodes within the network to discover multiple routes, at any point in time and use only one route. This is achieved by keeping a set of good routes and, a route for every packet choosing in a probabilistic fashion. Energyaware routing tries to increase network lifetime until the first node in the network dies (FND). Extending network lifetime ensures that energy is used equitably across the network until the death of the last node (LND). Energy resources within the network are optimally distributed and the individual nodes can survive for long run of time. Therefore, developing energy efficient routing protocols is considered to be taken in consideration in every aspect of design and operation.

In order to prolong the network lifetime, energy-efficient clustering protocols should be designed for the better characteristic of WSN. Efficiently organizing sensor nodes into clusters is useful in reducing energy consumption. Many energy-efficient routing protocols are proposed based on the clustering structure utilized for given structures [12, 13]. 
However, the LEACH algorithm which selects the cluster heads dynamically and frequently by round robin mechanism, which makes the cluster heads broadcast messages to all the sensor nodes in the network with additional energy consumption [14, 15]. DIS-RES-EL uses the initial and residual energy level of the nodes to select the cluster-heads. To avoid that each node needs to know the global knowledge of the networks, it estimates the ideal value of network lifetime, which is used to compute the reference energy that each node should expend during a round [16]. In RES-EL, the nodes with residual energy have more chance to be the cluster-heads than others [16].

In this paper, a new distributed energy-efficient clustering scheme for homogeneous wireless sensor networks, which is called IMP-EEL, had been proposed. In this algorithm, the cluster-heads are elected by a newly proposed energetic clustering probability based on the ratio between the residual energy of each node and the initial energy of the network. The nodes with high initial and residual energy will have more chances to be the cluster-heads than the low-energy nodes rotated in the epoch throughout the network. It had outdone RES-EL by extending network lifetime far better with new clustering strategies applied to it. Simulations show that the proposed algorithm achieves longer network lifetime, network stability, $\mathrm{CHs}$ formation, sending packets to both $\mathrm{BS}$ and $\mathrm{CHs}$ during its entire lifetime when compared with LEACH, DISRES-EL and RES-EL.

\section{THE PROPOSED ENRGY AWARE ROUTING PROTOCOL}

In an attempt to increase the strength of the lifetime of WSNs' in terms of cluster head selection, energy consumption, network lifetime and packet delivery from $\mathrm{CH}$ to $\mathrm{BS}$, the new probabilities elect cluster heads with the optimum routing established due to new clustering strategies[17].

\subsection{System Model and parameters}

Consider a wireless sensor network with $\mathrm{n}$ sensor nodes, $\mathrm{m}$ cluster heads and one base station. For convenience, each node is assigned a unique label as follows:

i. for each sensor node $i ; 1<i \leq n$,

ii. for each cluster node $j ; n<j \leq m+n$ and

iii. for the base station; $n+m+1$.

Consider a network of $n$ sensor nodes $1,2 \ldots n$ and a base station node $t$ labeled $n+1$ distributed over a region. The locations of the sensors and the base station are fixed and known a priori. Each sensor produces some information as it monitors its vicinity. It is assumed that each sensor generates one data packet per time unit to be transmitted to the base station. For simplicity, each time unit is referred as a round. It is assumed that all data packets have a size of $k$ bits. The information from all the sensors needs to be gathered at each round and forward to the base station for processing. The assumptions are:

i. Each sensor has the ability to transmit its packet to any other sensor in the network or directly to the base station.

ii. Each sensor $i$ has a battery with finite, nonrechargeable energy $E_{i}$.

iii. Whenever a sensor transmits or receives a data packet, it consumes some energy from its battery.

The base station has an unlimited amount of energy available to it.

\subsection{Improved Energy Efficient LEACH}

\subsubsection{Algorithm Details}

In IMP-EEL, clustering probabilities are modified against all the existing algorithms considered. Here, cluster heads are formed when residual energy level of each node is more than the remaining in epoch of the particular round.

The approach is to make it difficult for a node with low residual energy level to be selected as a cluster head [18]. If there is high residual energy at a node, the probability that it is selected as a cluster head increases by using new cluster head selection algorithm. The flowchart shown in Figure 1 gives the operation of the algorithm in detail.

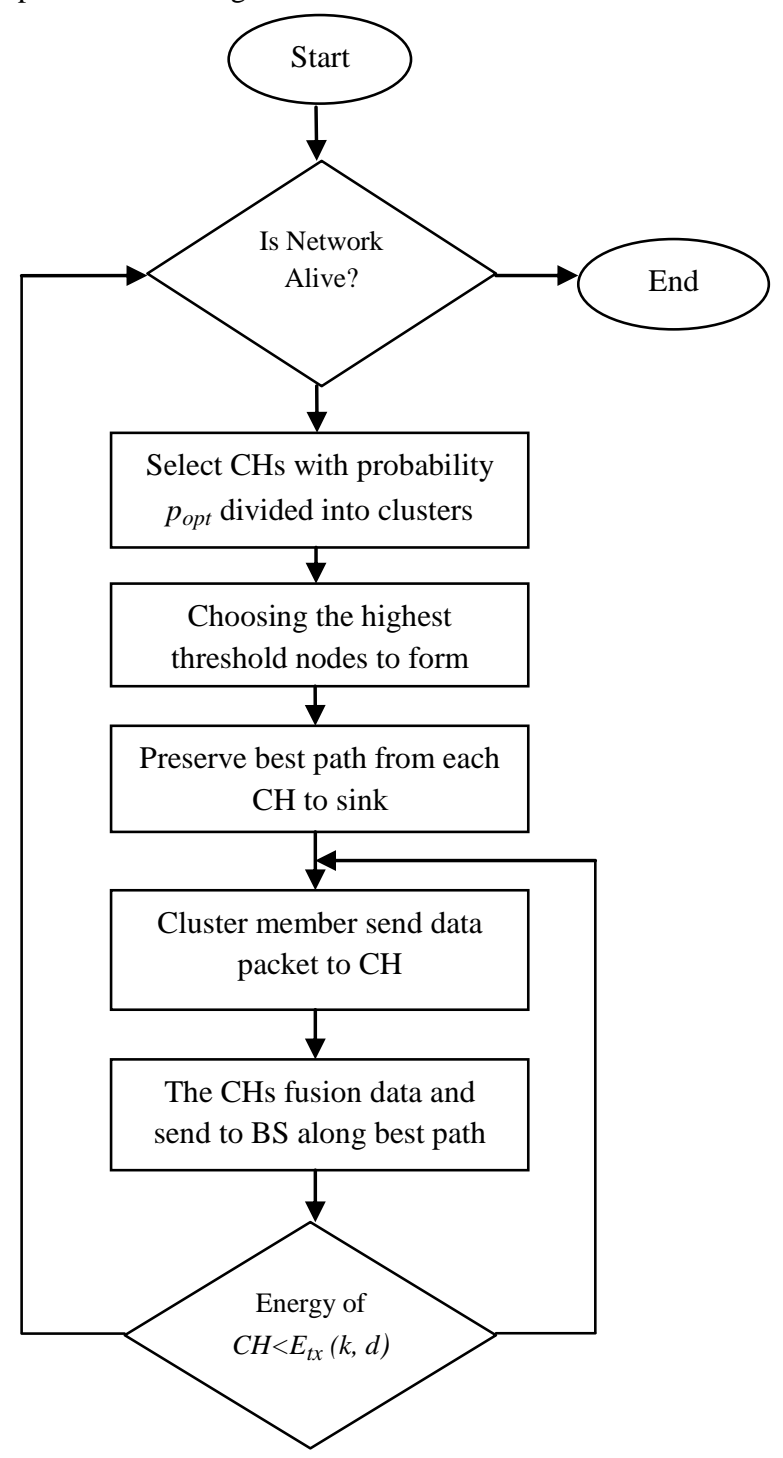

Fig 1: Flowchart of IMP-EEL

Let $n_{i}$ denote the number of rounds for the node $s_{i}$ to be a cluster head, and refer it as the rotating epoch. In homogeneous networks, to guarantee that there are average $p_{i} n$ cluster-heads in every round, let each node $s_{i}(i=1,2 \ldots n)$ become a cluster-head once every $n_{i}=1 / p_{i}$ rounds. The probability threshold each node $s_{i}$ uses to determine whether itself can become a cluster-head or not in each round, is given by

$$
T\left(s_{i}\right)=\frac{2 p_{i}}{1-p_{i}\left(\operatorname{rmod} \frac{1}{p_{i}}\right)} \frac{E_{\text {res }}}{E_{\text {init }}}, \quad s_{i} \in G
$$


where $G$ is the set of nodes that are eligible to become cluster heads at round $r$. If node $s_{i}$ has not been a cluster-head during the most recent $n_{i}$ rounds, then $s_{i} \in G$. In each round $r$, when node $s_{i}$ finds that it is eligible to be a cluster-head, it will choose a random number between 0 and 1 . If the number is less than threshold $T\left(s_{i}\right)$, the node $s_{i}$ becomes a cluster-head during the current round.

According to radio energy dissipation model [19] the energy expended to achieve signal to noise ratio in transmitting $k$-bit message over a distance $d$ is given as

$$
E_{T x(k, d)}=E_{\text {elec }} * k b i t+E_{f s} * k b i t * d^{2}
$$

where $E_{\text {elec }}$ is energy dissipated per bit to run transmitter or receiver circuit, $k b i t$ is the control packet length for every time, $E_{f s}$ depends on the transmitter amplifier model and $d$ is the distance between sender and receiver.

To receive a $k$-bit message the radio expends energy indicated

$$
E_{R x(k, d)}=E_{\text {elec }} * k b i t
$$

Assume an area over which ' $n$ ' nodes are uniformly distributed. Consider that sink is located at any place in the field, and distance of any node to the sink less than $d_{o}$ i.e...

$$
d_{o}=\sqrt{\left(E_{f s}\right) /\left(E_{m s}\right)}
$$

Data aggregation is the processing cost of a bit per report to sink. The energy transmission for non cluster head is obtained

$$
E=E_{\text {elec }} * k b i t+E_{f s} * k b i t * d^{2}
$$

$d=$ distance between sensor nodes and its cluster head, kbit $=$ packet length of sensor nodes.

Then the energy transmission for cluster head is given by

$$
E=E D A * \text { totalbit }+E_{f s} * k b i t * d^{2}
$$

totalbit $=$ packet length of $\mathrm{CH}$,

$d=$ distance from cluster head to node.

Let $d_{i, j}$ be the distance between nodes $i$ and $j$. Then, from (2) and (3), the total energy expenditure for delivering a $k$-bit data packet from node $i$ to node $j$ as follow:

$E_{i, j}=E_{T x}\left(k, d_{i, j}\right)+E_{R x}(k)=k\left\{2 E_{\text {elec }}+2 E_{f s}\left(d_{i, j}\right)^{2}\right\}$

Let $E_{\text {Total }}(i)$ be the total energy consumption of all the nodes in the WSN, i.e..,

$$
E_{\text {Total }}(i)=\sum_{i \in n} F_{\text {Total }}(i)
$$

Assume the initial energy of each node is equal to $E_{o} . F_{\text {Total }}(i)$ is the total energy expended by node $i$ for transmitting and receiving traffic, and $E_{\text {rem }}(i)$ the remaining energy of node $i$. So, remaining energy of a node after transmission and reception in the network is taken as [20]

$$
E_{\text {rem }}(i)=E_{o}-F_{\text {Total }}(i)
$$

According to (2) and (3) the energy consumed by node $i$ for receiving all the traffic from its neighbors is

$$
F_{R x}(i)=\sum_{j \in n b(i)} m_{j, i} \operatorname{Tr}(j) E_{\text {elec }}
$$

where $\operatorname{Tr}(j)$ the total traffic transmitted from node $j$.Whereas $m_{j, i}$ is the proportion of the traffic going through link $(j, i)$ to the total outgoing traffic at node $j$ and $n b(i)$ be the set of the neighbors of node $i$.

The energy expended by node $i$ for transmitting all the traffic to its neighbors is taken as

$$
F_{T x}(i)=\sum_{j \in n b(i)} m_{i, j} \operatorname{Tr}(i)\left\{E_{\text {elec }}+E_{f s} d_{i, j}^{2}\right\}
$$

where $\operatorname{Tr}(i)$ is the total traffic transmitted from node $i$, whereas $m_{i, j}$ is the proportion of the traffic going through link $(i, j)$ to the total outgoing traffic at node $i$. So, the full energy expenditure at node $i$ is given by [20]

$$
\begin{aligned}
& F_{\text {Total }}(i)=F_{R x}(i)+F_{T x}(i)= \\
& \sum_{j \in n b(i)} m_{j, i} \operatorname{Tr}(j) E_{\text {elec }}+\sum_{j \in n b(i)} m_{i, j} \operatorname{Tr}(i)\left\{E_{\text {elec }}+\right. \\
& \text { Efsdi,j2 }
\end{aligned}
$$

The optimal clustering created by this algorithm focuses the energy consumption and is well distributed over all the sensors. Total energy consumption is minimum compared to the other algorithms discussed earlier.

\section{IMPLEMENTATION AND RESULTS}

In this section, the performance of protocols is evaluated using MATLAB. Consider a wireless sensor network with $n=$ 100 nodes randomly distributed in a $100 \mathrm{~m} \times 100 \mathrm{~m}$ field. Without losing generalization, assume that the base station is in the center of the sensing region. To compare the performance of IMP-EEL with other protocols, ignore the effect caused by signal collision and interference in the wireless channel. The lifetime of the network is measured by the number of rounds until one node ceases functioning. In this situation, it is much more important to minimize the energy dissipation of the most heavily loaded nodes than to decrease the average energy dissipation.

The wireless transmission module can realize the transmitting power control or shut down automatically in order to avoid receiving unnecessary data according to the distance between the nodes. The radio parameters used in the simulations are shown in Table 1 [21]. The protocols compared are IMP-EEL include LEACH, DIS-RES-EL, and RES-EL.

Table 1. Parameters used in Simulations

\begin{tabular}{|l|l|}
\hline Parameter & Value \\
\hline Size of Network & $100 \mathrm{~m} \mathrm{X} 100 \mathrm{~m}$ \\
\hline Bandwidth & $1 \mathrm{Mb} / \mathrm{s}$ \\
\hline$E_{\text {elec }}($ Radio electronics energy) & $50 \mathrm{~nJ} / \mathrm{bit}$ \\
\hline$E_{\text {amp }}$ (Radio amplifier energy) & $100 \mathrm{pJ} / \mathrm{bit}^{\prime} \mathrm{m}^{2}$ \\
\hline$E_{\text {init }}$ (Initial energy of node) & $0.5 \mathrm{~J}$ \\
\hline Number of nodes & 100 \\
\hline Data Aggregation(EDA) & $0.5 \mathrm{~nJ} / \mathrm{bit}$ \\
\hline ctrPacket Length of EDA & 2000 bytes \\
\hline Packet length & 200 bytes \\
\hline
\end{tabular}


Here $p_{i}=0.05$, where $p_{i}$ is the probability to become cluster head per every round is considered.

The proposed algorithm is the best energy efficient algorithm to the homogeneous WSNs compared with LEACH, DISRES-EL and RES-EL algorithms with the following six issues considered:

(i). Total network lifetime until last node dies.

(ii). Network stability from starting round to death of the first node.

(iii). Total number of $\mathrm{CHs}$ formed during the entire lifetime of the algorithm. (iv). Aggregated data packets sent to $\mathrm{CHs}$ with their associated nodes in the cluster.

(v). Aggregated data packets sent to BS from the $\mathrm{CHs}$ during every round.

(vi). Percentage improvement of packets sent to BS from the $\mathrm{CHs}$ during lifetime of the network.

\subsection{Analysis of Results}

As shown in Figure 2, the network lifetimes of all the algorithms have been compared and the proposed algorithm had demonstrated better performance than the existing algorithms.

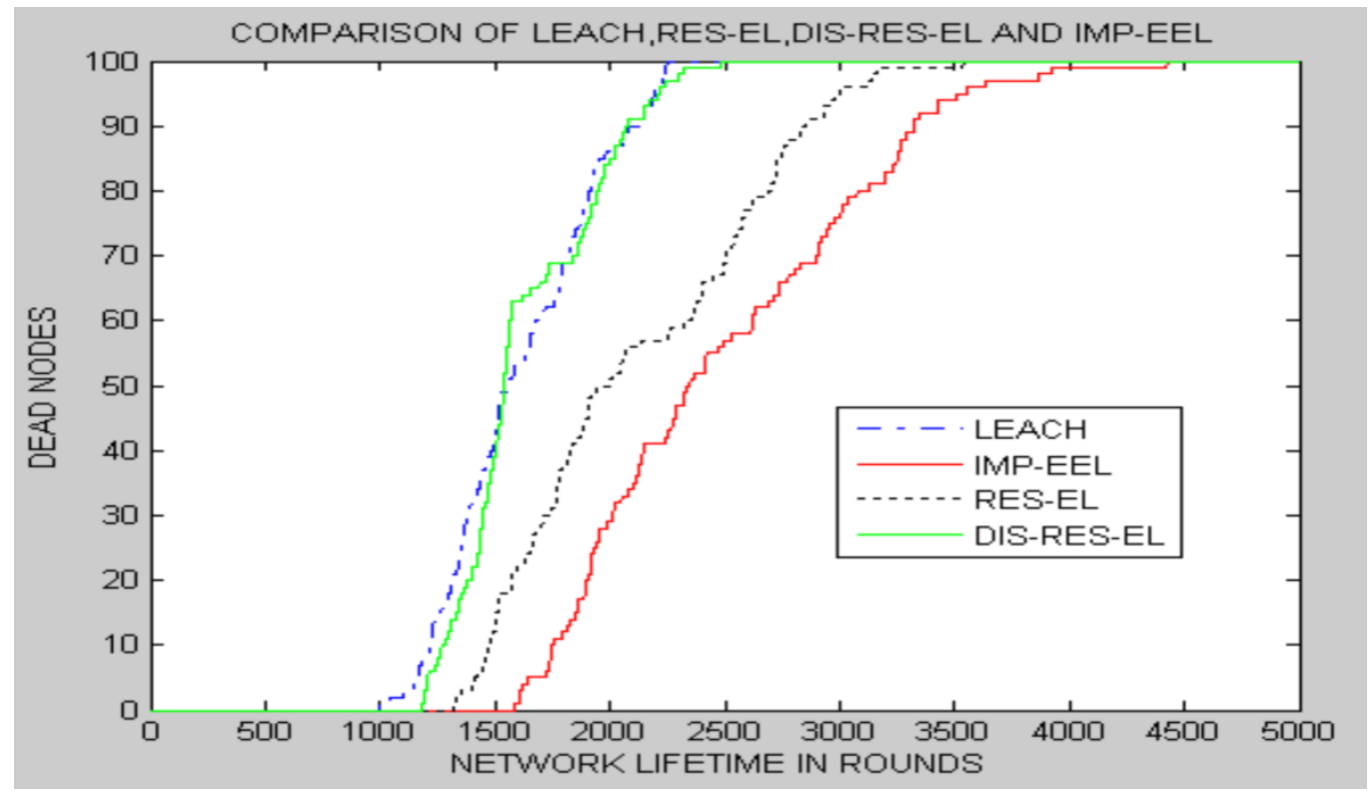

Fig 2: Comparison of network lifetime between all the algorithms

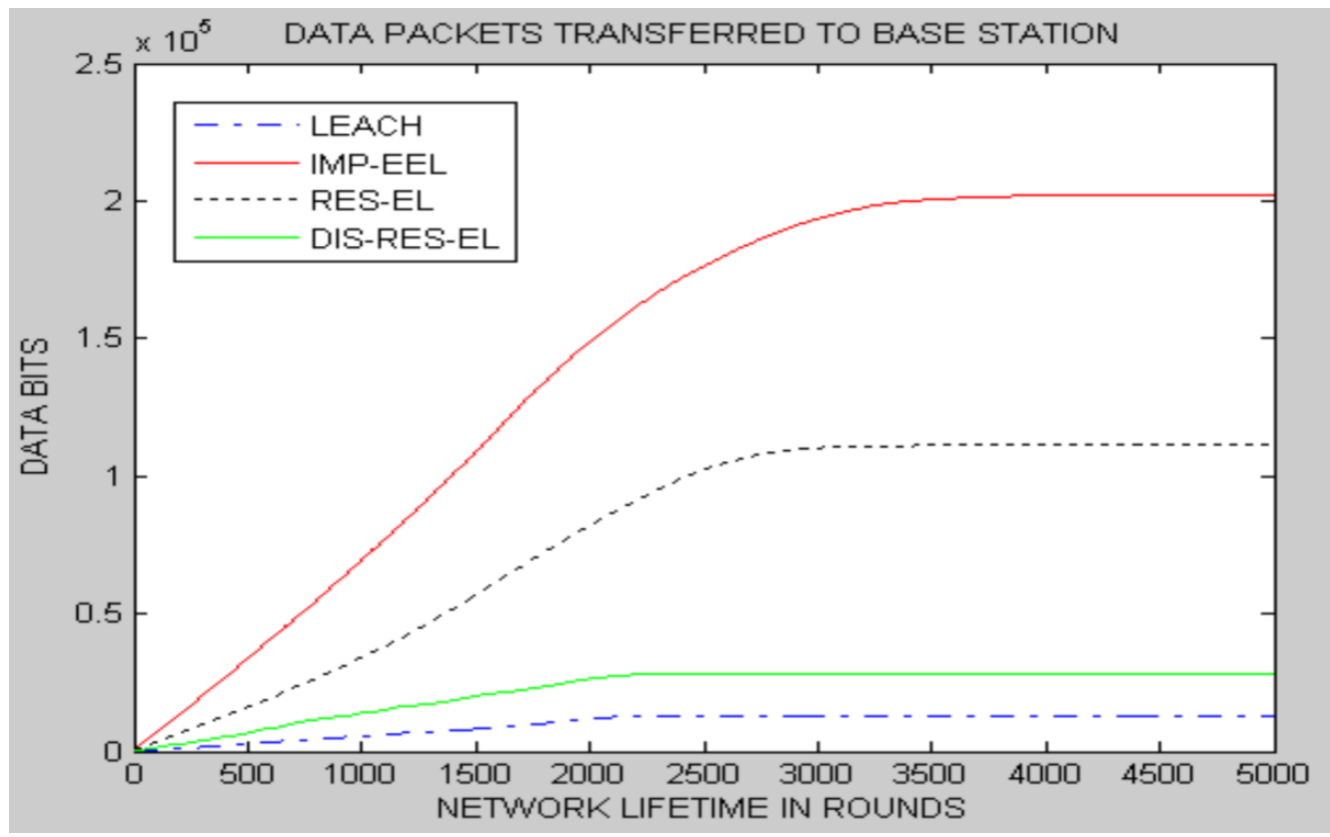

Fig 3: Comparison of aggregated data packets sent to BS between all the algorithms 
IMP-EEL had shown the improvement due the applied new clustering probability threshold proposed in Section III. The death of all the nodes in the proposed algorithm appeared at 4410 rounds while the same LEACH, DIS-RES-EL and RESEL had occurred at 2210, 2490 and 3530 rounds respectively. From this observation, the proposed algorithm has maintained the energy efficiency well than the remaining three algorithms.

While considering the above parameters in analyzing network lifetime from all the four algorithms, observations for the aggregated data packets travelling to both $\mathrm{BS}$ and $\mathrm{CH}$ had been noted. Figure 3 depicts the comparison of data packets sent to BS out of CHs from the above mentioned algorithms. From this result, IMP-EEL had sent more packets to BS than the remaining three algorithms. . It had transferred $2.02 \times 10^{5}$ data packets to BS from $2.5 \times 10^{5}$ data packets that had been received from their respective $\mathrm{CHs}$ during its entire lifetime. Where LEACH, DIS-RES-EL and RES-EL has transferred $1.3 \times 10^{4}, 2.8 \times 10^{4}$ and $1.1 \times 10^{5}$ data packets to BS from 1.48 $\times 10^{5}, 1.55 \times 10^{5}$ and $2.0 \times 10^{5}$ data packets that had been received from their respective $\mathrm{CHs}$ during their lifetime. Here

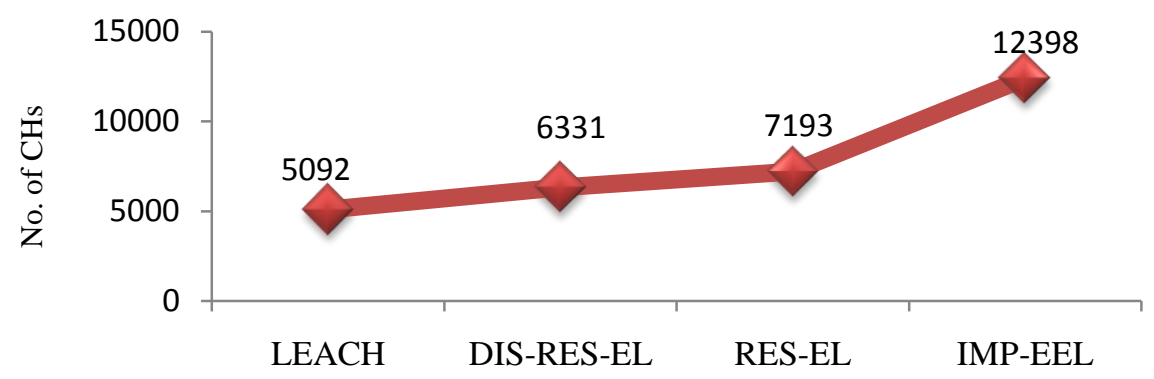

Comparison of $\mathrm{CHs}$ formation from all the algorithms

Fig 4: Comparison of formation of CHs during the entire lifetime from all the algorithms

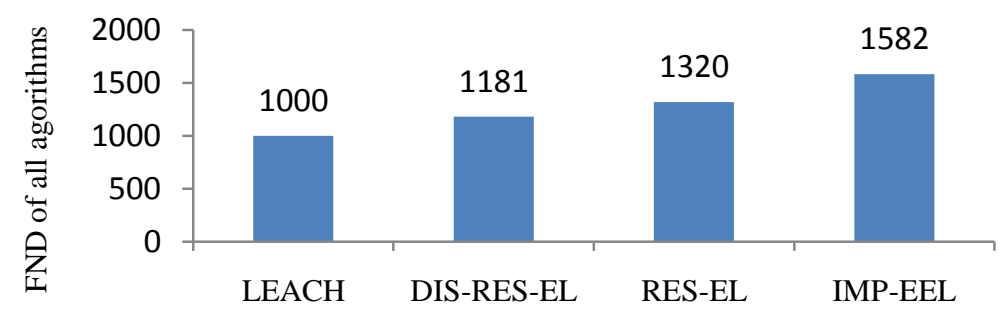

Comparison of network stability of all algorithms

Fig 5: Comparison of Network Stability from all the algorithms

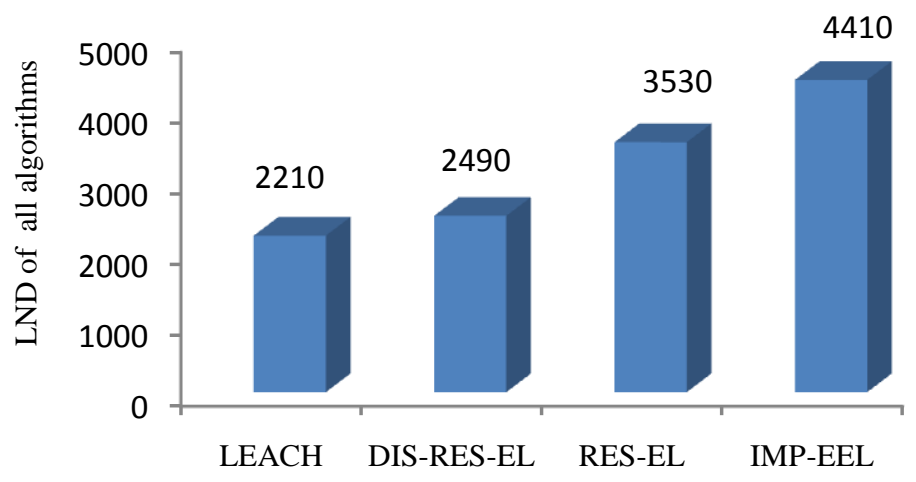

Comparison of network lifetime of all algorithms

Fig 6: Comparison of Network lifetime from all the algorithms 
also, IMP-EEL had shown improvement in transferring packets to BS with the other three algorithms.

Apart from the results shown in above Figures, the proposed algorithm had been compared with the other issues to evaluate its performance in energy efficiency nature including all six factors considered. The Figure 4 gives comparison of the total number of $\mathrm{CHs}$ formed in every round in their entire lifetime.
The Figure 5 depicts the comparison of network stability from first round to the death of first node (FND) for all the four algorithms. From the statistics shown below, the proposed algorithm maintained the network stability more than the other three algorithms due to newly proposed probability threshold value in selecting its CHs. IMP-EEL had occurred its FND at 1582 round which is far better when compared to LEACH, DIS-RES-EL and RES-EL.

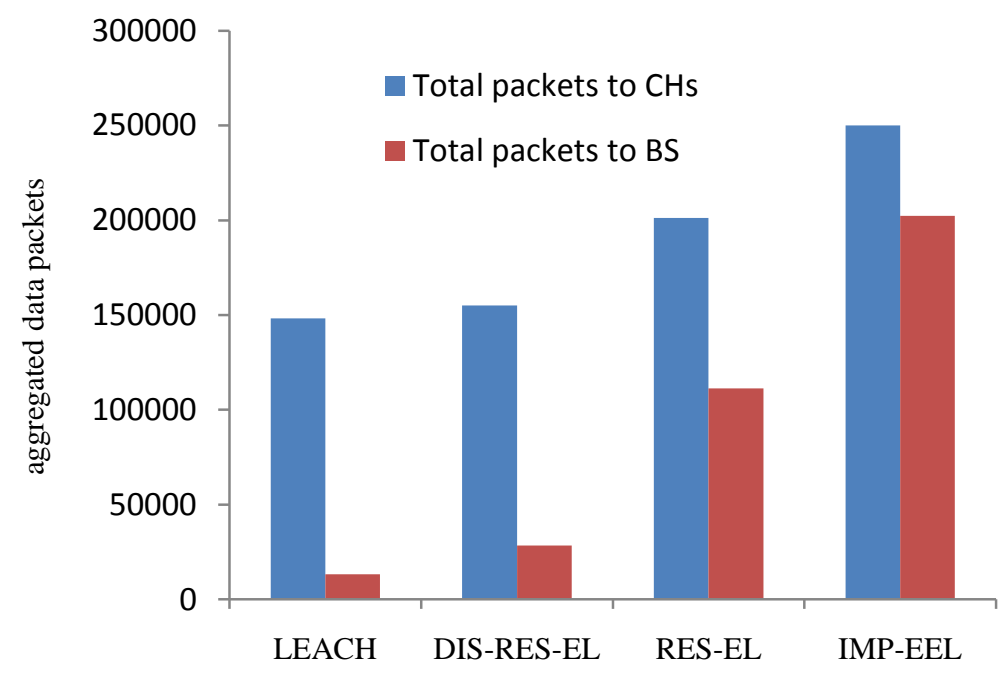

Comparison of packets sent to BS and to CHS from all algorithms

Fig 7: Comparison of aggregated packets sent to BS and CHs during their entire lifetime

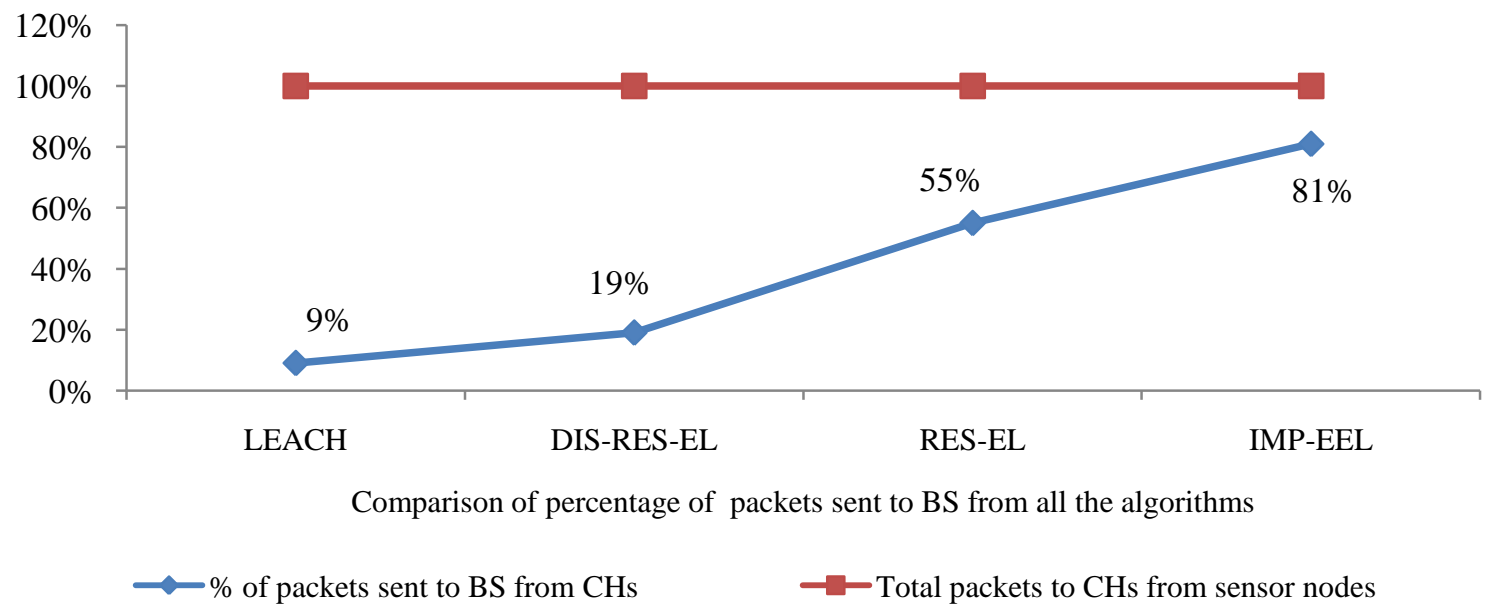

Fig 8: Percentage improvement in able to transfer packets to BS from CHs compared from all the algorithms

As shown in the Figure 4, IMP-EEL had $12398 \mathrm{CHs}$ compared to LEACH, DIS-RES-EL and RES-EL which have only 5092, 6331 and 7193 respectively in their entire lifetime. This shows that the proposed algorithm had improved its performance in selecting most energy efficient $\mathrm{CHs}$ in every round of the network.
In Figure 6, comparison to the network lifetime from all the four algorithms had been analyzed. The proposed algorithm had shown more energy efficiency than the other three as per the analysis shown in Figure 5. It indicates that with the new had able to extend its death of the last node (LND) further more from the remaining algorithms. 
Table 2. Comparison of all the algorithms with the features observed from the Simulations

\begin{tabular}{|l|l|l|l|l|l|c|}
\hline Algorithm & FND & LND & $\begin{array}{l}\text { Total CHs } \\
\text { formed }\end{array}$ & $\begin{array}{l}\text { Packets } \\
\text { sent to CHs }\end{array}$ & $\begin{array}{l}\text { Packets } \\
\text { sent to BS }\end{array}$ & $\begin{array}{l}\text { Percentage improvement of } \\
\text { packets sent to BS from CHs }\end{array}$ \\
\hline LEACH & 1000 & 2210 & 5092 & $1.4 \times 10^{5}$ & $1.3 \times 10^{4}$ & $9 \%$ \\
\hline DIS-RES-EL & 1181 & 2490 & 6331 & $1.5 \times 10^{5}$ & $2.8 \times 10^{4}$ & $55 \%$ \\
\hline RES-EL & 1320 & 3530 & 7193 & $2.01 \times 10^{5}$ & $1.11 \times 10^{5}$ & $81 \%$ \\
\hline IMP-EEL & 1582 & 4410 & 12398 & $2.5 \times 10^{5}$ & $2.02 \times 10^{5}$ & \\
\hline
\end{tabular}

The other important issue to be discussed from the four algorithms is the transferring of aggregated data packets travelling to $\mathrm{BS}$ and $\mathrm{CHs}$ involved in the simulations. As given in the statistics of Figure 7, the comparison of these algorithms in sending packets had been analyzed. From this analysis, it can be concluded that the proposed algorithm had been able to transfer both packets to BS from $\mathrm{CHs}$ and packets to $\mathrm{CHs}$ from sensor nodes more compared to the remaining three protocols. Hence, IMP-EEL had outdone in sending packets to both $\mathrm{BS}$ and $\mathrm{CHs}$ with the optimum routing achieved with the new algorithm.

Comparisons of percentage improvement between packets to $\mathrm{BS}$ and $\mathrm{CHs}$ are made for these four algorithms. The LEACH, DIS-RES-EL and RES-EL had 9\%, 19\% and 55\% respectively in sending packets to BS that had been received from their respective CHs. These three algorithms had not been able to transfer more packets from their $\mathrm{CHs}$ due to their clustering strategies being not effective in selecting more energetic $\mathrm{CHs}$ as their energy dissipates faster. While IMPEEL had shown tremendous improvement in selecting more energetic $\mathrm{CHs}$ during every round and also able to transfer more than the others as depicted in Figure 8.

The Table 2 gives the performances of all the four algorithms during their entire lifetime observed from the simulations with respect to the issues concerned above. Hence, from the statistics mentioned in the Table, IMP-EEL has $72 \%$ more energy efficiency than the conventional LEACH algorithm. It had outdone all the three remaining algorithms in all the aspects taken in consideration.

\section{CONCLUSIONS}

IMP-EEL routing protocol had been proposed in this paper. The proposed algorithm has more network lifetime, network stability, sending data packets to $\mathrm{BS}$ from respective $\mathrm{CHs}$ to sensor nodes and energy efficient $\mathrm{CHs}$ selection during their entire lifetime compared with LEACH, DIS-RES-EL and RES-EL. The simulation results confirm that with newly proposed clustering strategies with observation in each different scenario mentioned in the issues, IMP-EEL routing protocol as the most energy efficient algorithm of all the three algorithms for homogeneous WSNs.

\section{REFERENCES}

[1] Mohammad Ilyas and Imad Mahgoub. 2005. Handbook of Sensor Networks: Compact Wireless and Wired Sensing Systems. CRC Press LLC.
[2] W.R.Heinzelman. 2000. Application-Specific Protocol Architectures for Wireless Networks. Ph.D thesis, Massachusetts Institute of Technology.

[3] I Akyildiz, W. Su, Y Sankarasubramaniam, and E.Cayirci, "A survey on sensor networks", IEEE Communications Magazine (2002), 102-114.

[4] S. Tilak, N.B. Abu-Ghazaleh, and W. Heinzelman, "A taxonomy of wireless micro-sensor network models", ACM Mobile Computing Communications. Rev., 2002, 28-36.

[5] W.B. Heinzelman, A.P. Chandrakasan, and H. Balakrishnan, "An application-specific protocol architecture for wireless microsensor networks", IEEE Transactions on Wireless Communications (Oct. 2002), 660-670.

[6] J. Kulik, W. Heinzelman, and H. Balakrishnan, "Negotiation-based protocols for disseminating information in wireless sensor networks", Wireless Networks (March-May 2002), 169-185.

[7] A.B. McDonald and T.F. Znati, "A mobility-based framework for adaptive clustering in wireless adhoc networks", IEEE J. Selected Areas Communication. (Aug. 1999), 1466-1487.

[8] S.S. Pradhan, J. Kusuma, and K. Ramchandran, "Distributed compression in a dense microsensor network", IEEE Signal Processing. Mag. (Mar. 2002), 51-60.

[9] A. Scaglione and S. Servetto, "On the interdependence of routing and data compression in multihop sensor networks", In Proc. ACM Int. Conf. Mobile Computing. Networks (MobiCom'02), 2002, 140-147.

[10] C. Intanagowiwat, R. Govindan, and D. Estrin, "Directed diffusion: a scalable and robust communication paradigm for sensor networks", In ACM Int. Conf. Mobile Computing Networking (Mobi- Com'00), 2000, 56-67.

[11] Shah R.C. and Rabaey J.M, "Energy-aware routing for low energy ad hoc sensor networks", IEEE WCNC (2002), 350-355.

[12] P. Krishna, N.H. Vaidya, M. Chatterjee, D. Pradhan, "A cluster-based approach for routing in dynamic networks", ACM SIGCOMM Computer Communication Review (1997), 49-65. 
[13] B. McDonald, T. Znati. 2001. Design and performance of a distributed dynamic clustering algorithm for Ad-Hoc networks. In Proceedings of the Annual Simulation Symposium.

[14] Bandyopadhyay S, Coyle E, "An energy efficient hierarchical clustering algorithm for wireless sensor networks", In Proceedings of the 22nd annual joint conference of the IEEE computer and communications societies, 2003,1713-23.

[15] Liu JS, Lin $\mathrm{CH}$, "Power efficiency clustering method with power limit constraint for sensor networks performance", In Proceedings of the 2003 IEEE international performance, computing, and communications conference, 2003, 129-36.

[16] Li Qing, Qingxin Zhu, Mingwen Wang, "Design of a distributed energy-efficient clustering, algorithm for heterogeneous wireless sensor networks", Computer Communications (2006), 2230-2237.
[17] Ameer Ahmed Abbasi, Mohamed Younis, "A survey on clustering algorithms for wireless sensor networks", Computer Communications (2007), 2826-2841.

[18] Yan Zhang, Laurence T.Yang, and Jiming Chen. 2010. RFID and sensor networks: architectures, protocols, security, and integrations. CRC Press-Taylor \& Francis Group.

[19] A. Bari, A. Jaekel and S. Bandyopadhyay, "Maximizing the Lifetime of Two-Tiered Sensor Networks", In the Proceedings of IEEE International Electro/Information Technology Conference, 2006, 222-226.

[20] Yi-hua Zhu, Wan-deng, Wu Jian Pan, Yi-ping Tang, “An energy-efficient data gathering algorithm to prolong lifetime of wireless sensor networks", Computer Communications (2010), 639-647.

[21] WANG Guifeng, WAN,G Yong, Tao Xiaoling "An Ant Colony Clustering Routing Algorithm for Wireless Sensor Networks", IEEE Computer Society, 2009, 670673. 